\title{
Elastic Properties of Water and Ice VI Studied by Brillouin Scattering
}

\author{
H.Shimizu \\ Department of Electronics, Gifu University, 1-1 Yanagido, Gifu 501-11, Japan
}

\begin{abstract}
The pressure dependence of acoustic velocities and refractive index of liquid $\mathrm{H}_{2} \mathrm{O}$ and $\mathrm{D}_{2} \mathrm{O}$ have been determined up to 1.05 $\mathrm{GPa}$ at room temperature. For the tetragonal ice VI from $P=1.05$ to $2.10 \mathrm{GPa}$, we present the detailed method of the determination of six elastic constants, which are compared with the recent result at $0.72 \mathrm{GPa}$ and $271 \mathrm{~K}$ by Tulk et al. [ Brillouin scattering, elastic property, water, ice VI, elastic constant ]
\end{abstract}

\section{Introduction}

Water and ice are important materials in nature. Water can exist in many crystalline forms under different conditions of pressure and temperature. An interesting variety of these polymorphisms is mainly due to the hydrogen bond and the structural versatility of $\mathrm{H}_{2} \mathrm{O}$ molecules. At room temperature, compression of water leads to a tetragonal ice $\mathrm{VI}$ at pressures above $1.05 \mathrm{GPa}$ and further to a cubic ice VII above 2.10 GPa.

High-pressure Brillouin spectroscopy is a useful method to study elastic properties of molecular single crystals grown in a diamond anvil cell (DAC): Acoustic velocities for any direction, the refractive index, and adiabatic elastic constants can be exactly determined by only Brillouin scattering measurements with in situ identification of the crystal orientation at each pressure [1].

Polian and Grimsditch [2] made the Brillouin study only at a scattering geometry of $180^{\circ}$ and observed the pressure dependence of $n v$ in ice VI and VII phases without identification of the crystal orientation. Very recently, we have obtained the mapping of sound velocities for all directions in ice VI and VII (up to $8 \mathrm{GPa}$ ), and determined the pressure dependence of respective six and three elastic constants $[3,4]$.

In this paper, we present the pressure dependence of the acoustic velocity $(v)$ and the refractive index $(n)$ of liquid $\mathrm{H}_{2} \mathrm{O}$ and $\mathrm{D}_{2} \mathrm{O}$, and show the pressure dependence of 6 elastic constants of tetragonal $\mathrm{H}_{2} \mathrm{O}$ ice $\mathrm{VI}$ in the pressure range from 1.05 to $2.10 \mathrm{GPa}$ at $300 \mathrm{~K}$. These typical values of elastic constants are compared

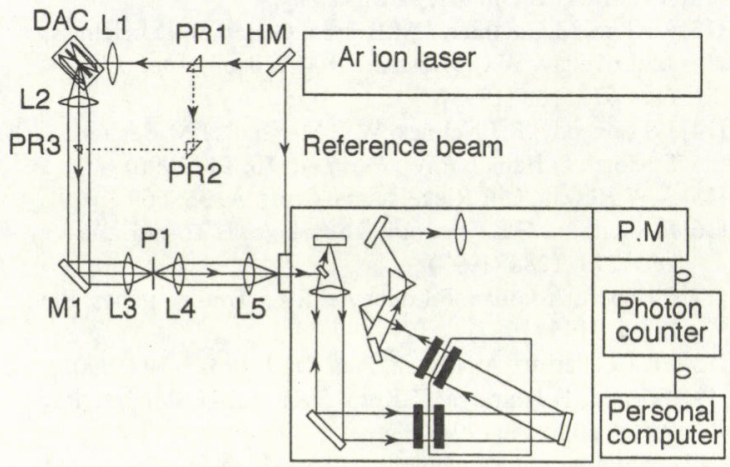

Tandem Fabry-Perot interferometer

Fig. 1. Experimental set up for $90^{\circ}$ Brillouin scattering under high pressure with DAC. PR1, PR2 and PR3 are used for $180^{\circ}$ scattering measurements. with those determined for ice VI at $0.72 \mathrm{GPa}$ and $271 \mathrm{~K}$ by Tulk $e t$ $a l$. [5], by concerning with the fitting method for the determination of 6 elastic constants.

\section{Experiment}

A Sandercock tandem Fabry-Perot interferometer was used in the triple-pass arrangement for Brillouin scattering measurements (see Fig. 1). The Brillouin frequency shifts $(\Delta v)$ at $90^{\circ}$ and $180^{\circ}$ scattering geometries with the DAC are related to the $v$ as follows;

$$
\Delta v_{90}=\sqrt{2} v_{90} / \lambda_{0} \quad(1), \quad \Delta v_{180}=(2 n) v_{180} / \lambda_{0}
$$

where $\lambda_{0}$ is a wavelength of the incident laser line, and $\Delta v_{90}$ is independent of the $n$. For the isotropic medium, $v_{90}$ is equal to $v_{180}$. Therefore, by using Eqs. (1) and (2), we can get

$$
\Delta v_{180} / \Delta v_{90}=\sqrt{2} n
$$

As a result, the refractive index $n$ can be determined by measuring $\Delta v_{180}$ and $\Delta v_{90}$

A single crystal of ice VI was grown by increasing the pressure on a seed crystal, which coexists with the water at about $1.05 \mathrm{GPa}$ at $300 \mathrm{~K}$. The seed crystal in the DAC presents sometimes a typical dipyramid shape as shown in Fig. 2. The (101) plane of the tetragonal $\mathrm{H}_{2} \mathrm{O}$ ice can be clearly seen and recognized, as indicated

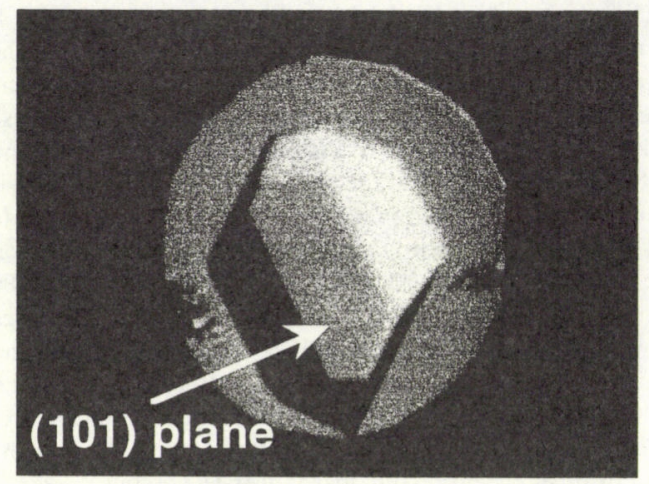

Fig. 2. Photograph of single crystal of ice VI coexisting with water at $1.05 \mathrm{GPa}$ and $300 \mathrm{~K}$. Crystal facets characterized by the tetragonal ice VI are clearly observed. 
previously by $x$-ray studies [6]. In order to study the elastic properties of ice VI, we used the single crystal grown from this type of seed [showing its (101) plane parallel to the culet faces of diamonds], because we need, as described later, the initial guess for the orientation of a single crystal in the DAC.

\section{Theoretical Basics}

From the secular equation for the propagation of elastic wave in a tetragonal system, Every [7] derived the relation between acoustic velocities for arbitrary direction and six elastic constants $\left(C_{11}, C_{12}\right.$, $\left.C_{13}, C_{33}, C_{44}, C_{66}\right)$. The acoustic velocities are expressed as a function of nine parameters as follows:

$$
v_{j}=g_{j}\left(C_{11} / \rho, C_{12} / \rho, C_{13} / \rho, C_{33} / \rho, C_{44} / \rho, C_{66} / \rho, \theta, \phi, \chi\right),
$$

where $\rho$ is density, the subscript $j(=0,1,2)$ of the velocity $v_{j}$ indicates $\mathrm{LA}, \mathrm{TA}_{1}$ (slow), and $\mathrm{TA}_{2}$ (fast) modes, respectively, and Euler angle $(\theta, \phi, \chi)$ relates the laboratory frame $(X, Y, Z)$ to the crystal reference frame $(x, y, z)$. In order to determine three Euler angles (= crystal orientation) and six elastic constants at each pressure, we applied a computerized least-squares fit between calculations $g_{j}\left(\phi_{i}\right)$ and experimental velocities $\left(\phi_{i}, v_{j i}\right)$ as a function of angle $\phi_{i}$,

$$
J=\sum_{i j}\left[g_{j}\left(\phi_{i}\right)-v_{j i}\right]^{2}, j=0,1,2
$$

where, after the set of initial values for nine parameters, $J$ is minimized by systematically varying the nine parameters until the fit is optimized. Then, according to the initial values set for nine parameters, we find two stability cases in which $J$ is minimized
(A)

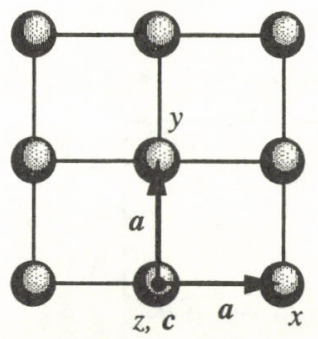

$\left[\begin{array}{cccccc}C_{11} & C_{12} & C_{13} & 0 & 0 & 0 \\ C_{12} & C_{11} & C_{13} & 0 & 0 & 0 \\ C_{13} & C_{13} & C_{33} & 0 & 0 & 0 \\ 0 & 0 & 0 & C_{44} & 0 & 0 \\ 0 & 0 & 0 & 0 & C_{44} & 0 \\ 0 & 0 & 0 & 0 & 0 & C_{66}\end{array}\right]$
(B)

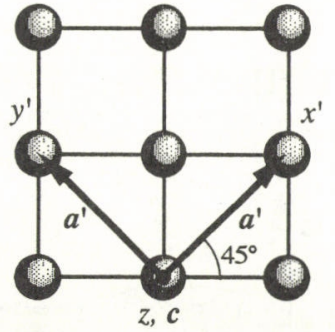

$\left[\begin{array}{cccccc}C_{11}^{\prime} & C_{12}^{\prime} & C_{13}^{\prime} & 0 & 0 & 0 \\ C_{12}^{\prime} & C_{11}^{\prime} & C_{13}^{\prime} & 0 & 0 & 0 \\ C_{13}^{\prime} & C_{13}^{\prime} & C_{33}^{\prime} & 0 & 0 & 0 \\ 0 & 0 & 0 & C_{44}^{\prime} & 0 & 0 \\ 0 & 0 & 0 & 0 & C_{44}^{\prime} & 0 \\ 0 & 0 & 0 & 0 & 0 & C_{66}^{\prime}\end{array}\right]$

$C_{11}^{\prime}=\left(C_{11}+C_{12}\right) / 2+C_{66}$

$C_{12}^{\prime}=\left(C_{11}+C_{12}\right) / 2-C_{66}$

$C_{13}^{\prime}=C_{13}, C_{33}^{\prime}=C_{33}, C_{44}^{\prime}=C_{44}$

$C_{66}^{\prime}=\left(C_{11}-C_{12}\right) / 2$
Fig. 3. Two cases (A) and (B) which are elastically equivalent each other for tetragonal lattice, and the corresponding matrix of elastic constants.

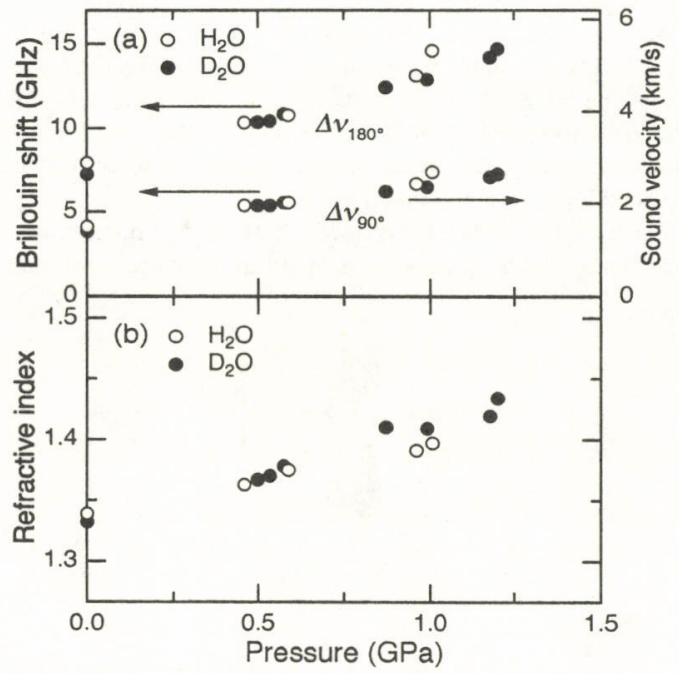

Fig. 4.(a) Pressure dependence of Brillouin frequency shifts at $90^{\circ}$ and $180^{\circ}$ scatterings for liquid $\mathrm{H}_{2} \mathrm{O}$ (open circles) and $\mathrm{D}_{2} \mathrm{O}$ (solid circles). Values of $90^{\circ}$ scattering indicate acoustic velocity simultaneously. (b) Pressure dependence of refractive indices $(n)$ for liquid $\mathrm{H}_{2} \mathrm{O}$ (open circles) and $\mathrm{D}_{2} \mathrm{O}$ (solid circles) at $300 \mathrm{~K}$.

with the optimum fit. Therefore, we should identify one true point, which is different from the cubic system $[1,3]$.

Let us investigate the origin of two stability points which appear on the least-squares fit for the tetragonal system. As shown in Fig. $3(\mathrm{~A})$, the tetragonal lattice is usually defined in a coordinate of $(x$, $y, z)$ - case A -. Then, the $(a, a, c)$ lattice indicates the matrix of adiabatic elastic constants as seen Fig. 3(A). However, another choice can be possible as shown in Fig. 3(B); $45^{\circ}$ rotated $\left(x^{\prime}, y^{\prime}, z\right)$ system about $z$-axis - case B -. This $\left(a^{\prime}, a^{\prime}, c\right)$ lattice is not crystallographycally identical to the $(a, a, c)$ lattice, but these two lattices can not be elastically distinguished. The matrix of elastic constants for $\left(a^{\prime}, a^{\prime}, c\right)$ lattice is easily derived as shown in Fig. 3(B). This is the reason why we find two (A and B) cases in which two stability points appear with the optimum fit. As a result, we need another information about the orientation of the tetragonal lattice in the DAC, in order to determine 6 elastic constants. As described in a later part, we used (101) or (101) plane of a dipyramid seed-crystal as the initial guess of the orientation of growing tetragonal $\mathrm{H}_{2} \mathrm{O}-\mathrm{VI}$ lattice.

\section{Results and Discussion}

For the liquid $\mathrm{H}_{2} \mathrm{O}$ and $\mathrm{D}_{2} \mathrm{O}$ at pressures up to about $1.2 \mathrm{GPa}$ and $300 \mathrm{~K}$, we determined the pressure dependence of $v$ and $n$. A high-pressure Brillouin spectrum of water at $90^{\circ}$ scattering geometry shows two signals: A strong peak indicates the $\Delta v_{90}$, which gives us the acoustic velocity by Eq.(1) as shown in Fig.4(a). The weak peak can be detected simultaneously as the $180^{\circ}$ scattering signal $\left(\Delta v_{180}\right)$, which is due to a laser beam reflected from the output diamond that serves as incident light. Figure 4(a) shows the pressure dependence of $\Delta v_{90}$ (sound velocity) and $\Delta v_{180}$ 
of liquid $\mathrm{H}_{2} \mathrm{O}$ and $\mathrm{D}_{2} \mathrm{O}$ below $1.2 \mathrm{GPa}$. Because the liquid is acoustically isotropic, the velocity is the same for any direction. Therefore, we determined the pressure dependence of $n$ by using Eq. (3). The $n$ of liquid $\mathrm{H}_{2} \mathrm{O}$ and $\mathrm{D}_{2} \mathrm{O}$, as seen in Fig. 4(b), show almost the same values and increase with pressure up to $n=1.41$ near the freezing point. The sound velocity and the refractive index show reasonable pressure dependence, and their values for liquid $\mathrm{H}_{2} \mathrm{O}$ and $\mathrm{D}_{2} \mathrm{O}$ are almost no difference.

For tetragonal $\mathrm{H}_{2} \mathrm{O}$ and $\mathrm{D}_{2} \mathrm{O}$ ice $\mathrm{VI}$, we present the method of determinations for the velocity mapping and 6 elastic constants as
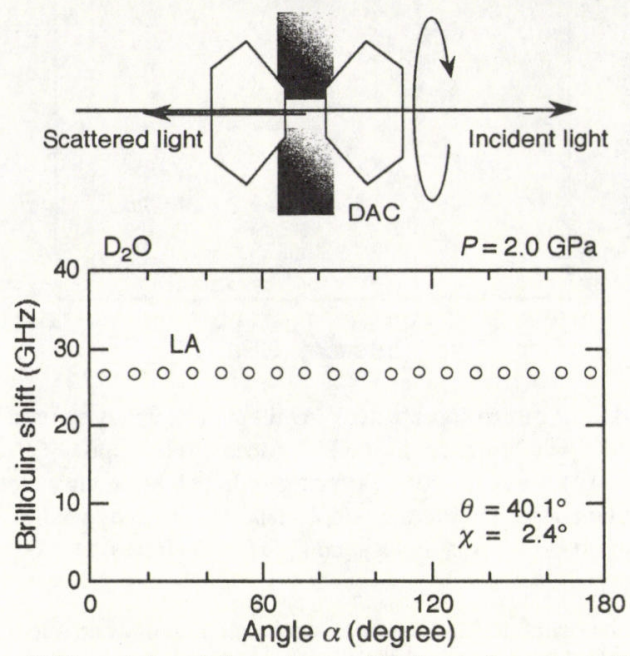

Fig. 5. The rotational dependence of Brillouin frequency shift at $180^{\circ}$ scattering geometry (solid $\mathrm{D}_{2} \mathrm{O}$ at $P=2.0 \mathrm{GPa}$ ). a function of pressure. Since the ice-VI crystal is usually birefringent, to estimate the anisotropic degree of refractive index, we measured the angular dependence of $\Delta v_{180}$ at $180^{\circ}$ scattering geometry [see Eq. (3)]. Since the velocity $v_{180}$ is independent of the rotation $(\phi)$ along the load axis of the DAC, we can estimate the direction dependence of $n$ by rotating the incident-light polarization (i.e., rotation of the DAC in $10^{\circ}$ intervals as shown in Fig. 5). The $\phi$ dependence of $\Delta v_{180}$ for the LA mode was measured at $P=2.0 \mathrm{GPa}$ as shown in Fig. 5. The $\Delta v_{180}$ is almost independent of $\phi$, that is, $\Delta v_{180}=26.85 \pm 0.135 \mathrm{GHz}$. This result indicates that the birefringence is less than about $1 \%$, which agrees with the previous study [2] by measuring Brillouin shifts with two cases of the incident light polarized parallel and perpendicular to the crystal axes at $180^{\circ}$ scattering geometry. Therefore, from our analysis of sound velocity, we can neglect the anisotropy of the refractive index in the ice VI within the experimental error on the measurements of Brillouin frequency shifts.

Brillouin measurements were made at $90^{\circ}$ scattering geometry in $10^{\circ}$ intervals of the rotation angle $\phi$ about the load axis of the DAC. The observed Brillouin shifts, that is, sound velocities for $\mathrm{H}_{2} \mathrm{O}$ ice at $1.23 \mathrm{GPa}$ [from Eq. (1)], are plotted as a function of $\phi$ by open symbols in Fig. 6 . To analyze the angular dependence of velocities of the $\mathrm{LA}$ and the two $\mathrm{TA}_{1}$ (slow) and $\mathrm{TA}_{2}$ (fast) modes at each applied pressure, we used Eq. (4). The computerized leastsquares fit was applied to determine the elastic constants and Euler angles (orientation of a $\mathrm{H}_{2} \mathrm{O}$ single crystal grown in the DAC). The best-fit calculations are represented by the solid lines in Fig. 6 for different initial sets of parameter values. Therefore, we met two cases in which $J$ (Eq. 5) was minimized with the optimum fit. In Fig. 6 we can see the final values of fitted 8 parameters and the error of least-squares fit (lse) for two cases. How do we select the true one from two cases? As mentioned above, we grew up the ice
(A)

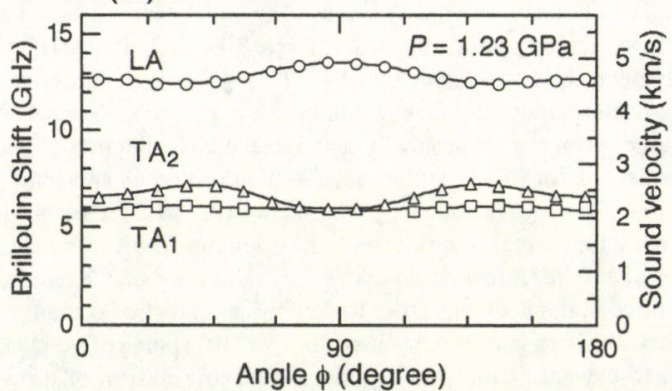

fitted parameters and the error of fitting

$$
\begin{aligned}
& \begin{array}{cccccc}
C_{11} / \rho & C_{12} / \rho & C_{13} / \rho & C_{33} / \rho & C_{44} / \rho & C_{66} / \rho \\
\hline 24.1 & 8.8 & 10.8 & 20.3 & 4.6 & 4.3
\end{array} \\
& \begin{array}{cc}
\theta \quad \chi & \text { 1se } \\
\hline 43.7 \quad 1.4 & \frac{122}{0.122}
\end{array}
\end{aligned}
$$

(B)

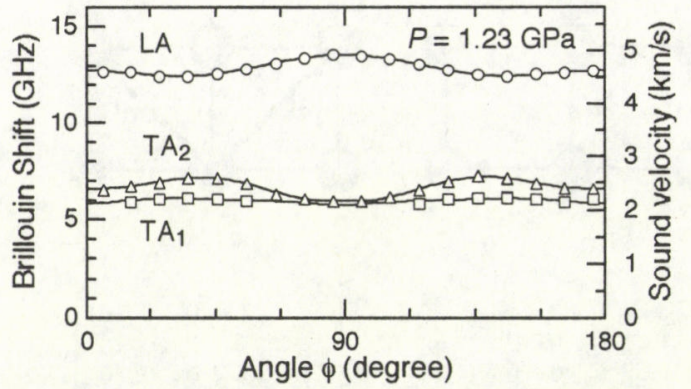

fitted parameters and the error of fitting

$$
\begin{array}{cccccc}
C_{11} / \rho & C_{12} / \rho & C_{13} / \rho & C_{33} / \rho & C_{44} / \rho & C_{66} / \rho \\
\hline 20.8 & 12.2 & 10.8 & 20.3 & 4.6 & 7.7
\end{array}
$$$$
\text { calculation } \longrightarrow \text { (20.8) (12.2) (10.8) (20.3) }
$$

from case (A)

Fig. 6. Brillouin frequency shifts and acoustic velocities of $\mathrm{LA}, \mathrm{TA}_{2}$, and $\mathrm{TA}_{1}$ modes as a function of angle $\phi$ at a $90^{\circ} \mathrm{scattering}$ geometry for $\mathrm{H}_{2} \mathrm{O}$ ice $\mathrm{VI}$ at $P=1.23 \mathrm{GPa}$. Open symbols indicate experimental points, and the solid lines represent the fitted velocities. For cases (A) and (B), fitted parameters are indicated with the error of fitting (lse). The parentheses for case (B) shows the calculated values from case (A) by the use of relation in Fig. 3. 


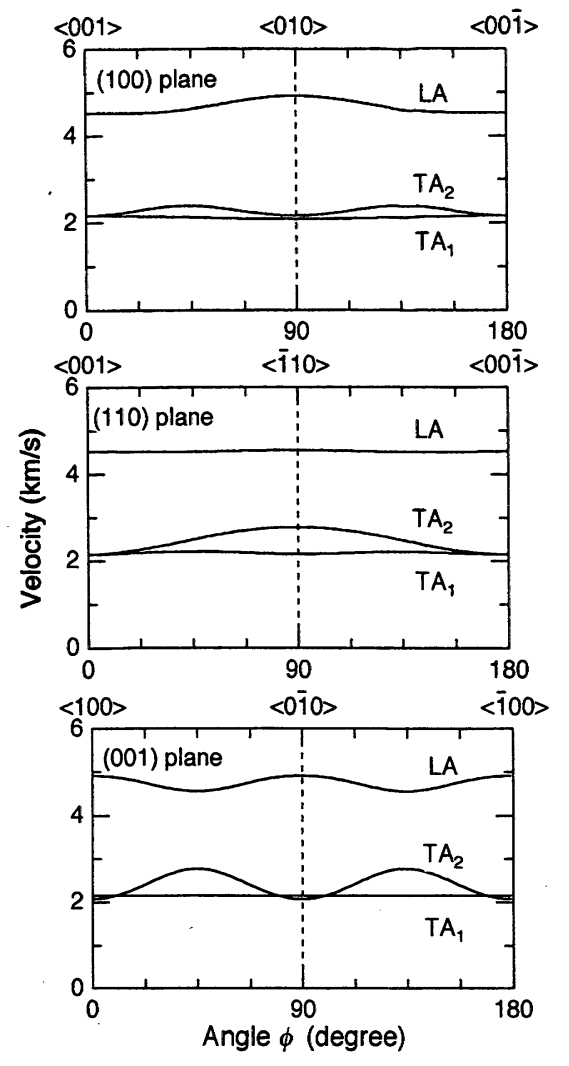

Fig. 7. Velocity mapping in typical planes for $\mathrm{H}_{2} \mathrm{O}$ ice $\mathrm{VI}$ at $P=1.23 \mathrm{GPa}$ and $300 \mathrm{~K}$.

VI single-crystal showing its (101) or (101) plane parallel to the culet faces of diamonds. Therefore, this situation indicates that the Euler angle $\chi$ should be nearly $0^{\circ}$ or $90^{\circ}$. As seen in Fig. 6 , case (A) shows $\chi=1.4^{\circ}$, and case (B) does $\chi=46.4^{\circ}$. As a result, case (A) yielded the perfect solution of $C_{11} / \rho=24.1 \mathrm{~km}^{2} \mathrm{~s}^{-2}, C_{12} /$ $\rho=8.8 \mathrm{~km}^{2} \mathrm{~s}^{-2}, C_{13} / \rho=10.8 \mathrm{~km}^{2} \mathrm{~s}^{-2}, C_{33} / \rho=20.3 \mathrm{~km}^{2} \mathrm{~s}^{-2}, C_{44} / \rho=$ $4.6 \mathrm{~km}^{2} \mathrm{~s}^{-2}, C_{66} / \rho=4.3 \mathrm{~km}^{2} \mathrm{~s}^{-2}$ and the crystal orientation $\theta=$ $43.7^{\circ}$ and $\chi=1.4^{\circ}$ within an accuracy of $\pm 2 \%$. Here, it is noted that the $\chi=46.4^{\circ}\left(=45^{\circ}+1.4^{\circ}\right)$ for case $(B)$ indicates the $45^{\circ}$ rotation of case (A) around $z$-axis. From the relation of $C_{11}^{\prime}=$ $\left(C_{11}+C_{12}\right) / 2+C_{66}, C_{12}^{\prime}=\left(C_{11}+C_{12}\right) / 2-C_{66}, C_{13}^{\prime}=C_{13}, C_{33}^{\prime}=$ $C_{33}, C_{44}^{\prime}=C_{44}$, and $C_{66}^{\prime}=\left(C_{11}-C_{12}\right) / 2$ stated in Fig. 3 , the parameter values are calculated, and are shown in parentheses at case (B) in Fig. 6 . The agreement between this calculated value and the fitted one is very good.

Once the nine parameters were determined, the acoustic velocities [ $v=\left(C_{\mathrm{ij}} / \rho\right)^{1 / 2}$ ] could be calculated for all directions. The typical velocity mapping of ice VI at $P=1.23 \mathrm{GPa}$ is shown in Fig. 7 . Next, we show the pressure dependence of acoustic velocities in typical $<100>$ and $<110>$ directions of $\mathrm{H}_{2} \mathrm{O}$ and $\mathrm{D}_{2} \mathrm{O}$ ice VI in Fig. 8. The acoustic velocity in a liquid shows discontinuous changes to

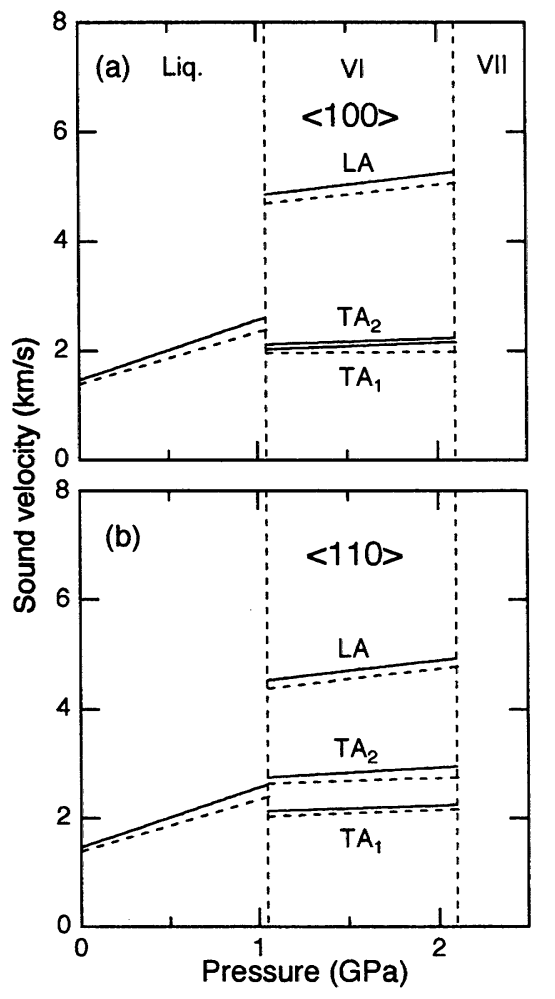

Fig. 8. The pressure dependence of acoustic velocities for (a) $<100>$ and $(b)<110>$ directions at $300 \mathrm{~K}$ in tetragonal $\mathrm{H}_{2} \mathrm{O}$ (solid line) and $\mathrm{D}_{2} \mathrm{O}$ (broken line) ice up to $2.10 \mathrm{GPa}$. The acoustic velocity in liquid $\mathrm{H}_{2} \mathrm{O}$ and $\mathrm{D}_{2} \mathrm{O}$ show discontinuous changes to three acoustic modes at the freezing pressure of $P=1.05 \mathrm{GPa}$.

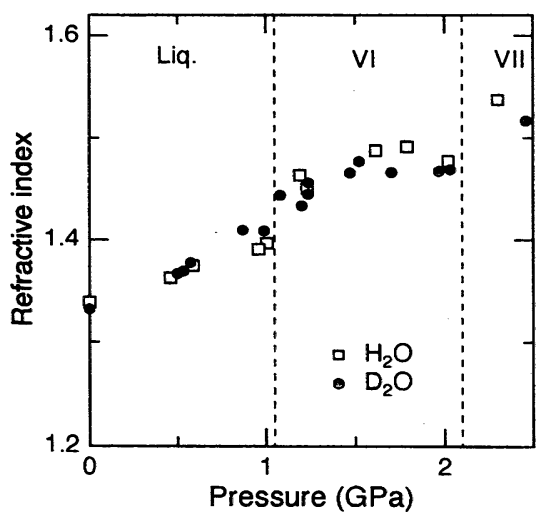

Fig. 9. Pressure dependence of refractive index at $300 \mathrm{~K}$ in a liquid water up to $1.05 \mathrm{GPa}$ and tetragonal ice VI up to $2.1 \mathrm{GPa}$. Open squares indicate $\mathrm{H}_{2} \mathrm{O}$ and solid circles do $\mathrm{D}_{2} \mathrm{O}$. 


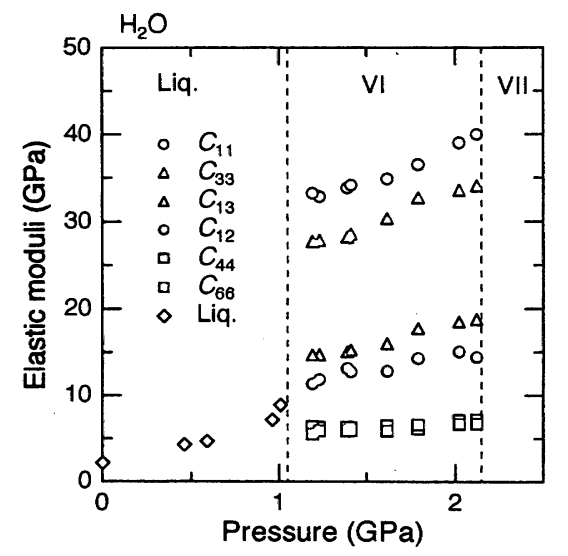

Fig. 10. Pressure dependence of elastic moduli in a liquid $\mathrm{H}_{2} \mathrm{O}(C=\rho v 2)$ up to $1.05 \mathrm{GPa}$ and tetragonal $\mathrm{H}_{2} \mathrm{O}$ ice VI $\left(C_{11}\right.$, $C_{12}, C_{13}, C_{33}, C_{44}$, and $\left.C_{66}\right)$ up to $2.10 \mathrm{GPa}$ at $300 \mathrm{~K}$.

$\mathrm{LA}, \mathrm{TA}_{2}$ and $\mathrm{TA}_{1}$ in ice $\mathrm{VI}$, and they increase with pressure. The distinction in acoustic velocity for $\mathrm{H}_{2} \mathrm{O}$ ice $\left(v_{\mathrm{H}_{2}} \mathrm{O}\right)$ and $\mathrm{D}_{2} \mathrm{O}$ ice $\left(v_{\mathrm{D}_{2} \mathrm{O}} \mathrm{O}\right.$ is understood by the kinetic theory, $v_{\mathrm{H}_{2}} \mathrm{O} v_{\mathrm{D}_{2} \mathrm{O}}=\left(M_{\mathrm{D}_{2} \mathrm{O}} M_{\mathrm{H}_{2} \mathrm{O}}\right)^{1 / 2}=$ 1.054 , where $M_{\mathrm{D}_{2} \mathrm{O}}$ and $M_{\mathrm{H}_{2} \mathrm{O}}$ are the molecular weight of $\mathrm{D}_{2} \mathrm{O}$ and $\mathrm{H}_{2} \mathrm{O}$, respectively. This relation is approximately obeyed at each pressure.

Furthermore, we can calculate the acoustic velocity $\left(v_{180}\right)$ along the direction perpendicular to diamond interfaces, which is available to determine the refractive index $(n)$ by using Eq. (3). From the $\Delta v_{180}$ measured at $180^{\circ}$ scattering geometry, we can obtain the pressure dependence of $n$ for $\mathrm{H}_{2} \mathrm{O}$ and $\mathrm{D}_{2} \mathrm{O}$ ice $\mathrm{VI}$ as shown in Fig.9. The uncertainties in the measured frequency shifts and in the determined refractive index are about $\Delta(\Delta v) / \Delta v= \pm 1 \%$ and $\Delta n / n=$ $\pm 2 \%$, respectively. At the freezing point, $n$ shows a discontinuous increase of about $4 \%$ and, moreover, increases gradually with pressure up to $n=1.48$ at $P=2.10 \mathrm{GPa}$. The $n$ of $\mathrm{H}_{2} \mathrm{O}$ ice shows almost the same values as $\mathrm{D}_{2} \mathrm{O}$ ice, and the present results are in good agreement with those estimated by Polian and Grimsditch [2].

In Fig. 10 we show six elastic constants for $\mathrm{H}_{2} \mathrm{O}$ ice $\mathrm{VI}$ as a function of pressure between 1.05 and $2.10 \mathrm{GPa}$ by using the pressure dependence of $\rho$ from X-ray studies [8]. In Table 1 our
Table 1. Elastic moduli (GPa) in tetragonal $\mathrm{H}_{2} \mathrm{O}$ ice VI determined by the present study and Tulk et al.[5], and the calculated values by operating a $45^{\circ}$ rotation of crystalline axes about $z$-axis for the system of Tulk et al.

\begin{tabular}{|c|c|c|c|c|c|c|}
\hline & \multicolumn{6}{|c|}{ Elastic moduli (GPa) } \\
\hline & $C_{11}$ & $C_{12}$ & $C_{13}$ & $C_{33}$ & $C_{44}$ & $C_{66}$ \\
\hline $\begin{array}{l}\text { Present study } \\
(P=1.23 \mathrm{GPa}, T=300 \mathrm{~K})\end{array}$ & 32.8 & 11.8 & 14.7 & 27.8 & 6.3 & 5.9 \\
\hline $\begin{array}{l}\text { Tulk et al. }[5] \\
(P=0.72 \mathrm{GPa}, T=271 \mathrm{~K})\end{array}$ & 26.8 & 14.5 & 12.8 & 26.2 & 6.3 & 10.4 \\
\hline $\begin{array}{l}45^{\circ} \text { rotation around } \\
z \text {-axis for Tulk et al. }\end{array}$ & 31.1 & 10.3 & 12.8 & 26.2 & 6.3 & 6.1 \\
\hline
\end{tabular}

typical values of $P=1.23 \mathrm{GPa}$ are listed with recent results of ice $\mathrm{VI}$ at $0.72 \mathrm{GPa}$ and $271 \mathrm{~K}$ by Tulk et al. [5]. The difference exists in their relative values and in their inequality of 6 elastic constants: present results; $C_{11}>C_{33}, C_{12}<C_{13}, C_{44}>C_{66}$, Tulk et al.; $C_{11} \approx$ $C_{33}, C_{12}>C_{13}, C_{44}<C_{66}$. If we operate a $45^{\circ}$ rotation of crystalline axes around $z$-axis for the system of Tulk et al., the calculated values which are very close to our present results were obtained as listed in Table 1. Tulk et al. used a large high-pressure cell made from a steel block, $7 \times 7 \times 10 \mathrm{~cm}$, and they obtained the initial guess of two Euler angles, by using the extinction method of polarized light due to birefringence of ice VI [5]. (birefringence is less than about $1 \%$ )

\section{Acknowledgment}

I would like to thank my colleagues Assoc. Prof. S. Sasaki, Dr. T. Kume, and T. Nishiba (now, Koito Corp., Shizuoka) for their contribution to this study.

\section{References}

[1] H. Shimizu, S. Sasaki, Science 257, 514 (1992).

[2] A. Polian, M. Grimsditch, Phys. Rev., B 27, 6409 (1983).

[3] H. Shimizu et al., Phys. Rev. Lett., 74, 2820 (1995).

[4] H. Shimizu et al., Phys. Rev., B 53, 6107 (1996).

[5] C. A. Tulk et al., J. Chem. Phys., 104, 7854 (1996).

[6] K. Yamamoto, Jpn. J. Appl. Phys., 21, 803 (1982).

[7] A. G. Every, Phys. Rev., B 22, 1746 (1980).

[8] R. G. Munro et al., J. Appl. Phys., 53, 6174 (1982). 